\title{
Visible light Terrestrial Planet Finder: planet detection and spectroscopy by nulling interferometry with a single aperture telescope
}

Bruce Martin Levine, Michael Shao, Charles A. Beichman, Bertrand P. Mennesson, Rhonda M. Morgan, et al.

Bruce Martin Levine, Michael Shao, Charles A. Beichman, Bertrand P. Mennesson, Rhonda M. Morgan, Glenn S. Orton, Eugene Serabyn, Stephen C. Unwin, T. Velusamy, Neville J. Woolf, "Visible light Terrestrial Planet Finder: planet detection and spectroscopy by nulling interferometry with a single aperture telescope," Proc. SPIE 4852, Interferometry in Space, (26 February 2003); doi: 10.1117/12.460925

SPIE Event: Astronomical Telescopes and Instrumentation, 2002, Waikoloa, Hawai'i, United States 


\title{
A Visible Light Terrestrial Planet Finder --Planet Detection and Spectroscopy by Nulling
} Interferometry with a Single Aperture Telescope

\author{
B.M. Levine, Michael Shao, C.A. Beichman, B. Mennesson, R. Morgan, G. Orton, \\ E. Serabyn, S. Unwin, T. Velusamy, \\ Jet Propulsion Laboratory/California Institute of Technology \\ 4800 Oak Grove Drive \\ Pasadena, CA 91109-8099
}

and

\author{
N. Woolf \\ University of Arizona, Seward Observatory, Tucson, AZ
}

\section{Keywords: Planet Detection, Nulling Interferometer, Coronagraph, Space Telescope}

\section{Abstract}

Planet detection around a bright star is dependent on the resolution of the imaging system and the degree of light suppression of the star relative to the planet. We present a concept and a scaled precursor for a visible light Terrestrial Planet Finding (VTPF) mission. Its major feature is an imaging system for planet detection using a nulling interferometer behind a single aperture telescope. This configuration is capable of detecting earth-like planets with a $4 \mathrm{~m}$ aperture using both imaging and spectroscopic imaging modes. We will describe the principles of the system, and show results of studies demonstrating its feasibility.

\section{Introduction}

The detection of extrasolar planets does not, in principle, require large collecting areas for either resolution or photometric purposes. If we view our solar system from 10 parsec in the visible, a Jupiter-like planet has an approximate magnitude of $V \sim 27$ and the Earth is $V \sim 30$. The Hubble Space Telescope $(D=2.4 \mathrm{~m})$ can detect a $V=30$ object, so a 27 magnitude object takes much less than $1 \mathrm{hr}$ of integration. In terms of resolution the orbit of a Jupiter-like planet at 10 parsec subtends an angle of 0.5 arc seconds, which requires a diffraction limited telescope of only $30 \mathrm{~cm}$ or greater (at $0.75 \mu \mathrm{m}$ wavelength) .

With a flux ratio in the optical of $\sim 10^{-9}-10^{-10}$ between a planet and its star, the harder problem is that of contrast. Achieving a very low background against which to detect a planet requires control of both scattered and diffracted light. Adaptive optics (AO) coronagraphs (Malbet, Yu, and Shao, 1995) provide a partial solution, by using a deformable mirror to improve the wavefront quality and by creating a 'dark hole' at the center of the field of view, effectively improving the telescope Strehl. Effectively, the deformable mirror reduces the scattering or mid spatial frequency errors that cause spurious scattering into the region where the planets would be found. In the recent studies for TPF, four teams studied numerous concepts for direct detection of planets including coronagraphs and apodized aperture telescopes. For the detection of an Earth-like planet in the visible, around a Sun at 10pc, a large telescope ( 8-10m diameter) was recommended to place the planet at least 3-4 Airy rings from the star. The size of the aperture is needed to spatially separate the star from the planet but is unnecessary for the fundamental task of collecting photons from the planet. A nulling interferometer, however, can be used to suppress both diffraction and scattering, and it can be located behind a much smaller aperture to resolve an extrasolar planet. A nulling interferometer effectively cancels the starlight and has $100 \%$ transmission for planet light when the optical path from the planet is $\lambda / 2$ different from the star. For our concept this corresponds to $\theta=0, \lambda / b, 3 \lambda / b$, ( $\mathrm{b}=$ interferometer baseline). By contrast a coronagraph operates out at several Airy rings $(\sim 3-4 \times 1.22 \lambda / D)$. For a modest sized aperture, about $\mathrm{D}=1.5 \mathrm{~m}$, a Jupiter-like planet could be resolved by synthesizing an interferometer with a $30 \mathrm{~cm}$ baseline.

This paper describes a new instrument for direct planet detection. It synthesizes a four element nulling interferometer from the telescope pupil to suppress the diffraction from a central star. After nulling, an array of coherent single mode optical fibers is used to negate the effects of residual stellar leakage (scattering) due to imperfections in the telescope optics and optical train. A simple imaging system after this array forms the final extrasolar planet image. This concept combines all the advantages of a nulling interferometer with the simplicity of a modest size and modest optical quality single aperture telescope. Such a telescope with this nulling interferometer as back-end instrument can image and detect planets, or provide the input to a low resolution spectrometer. Its primary optic is at least two times smaller in diameter (4 times in area) proposed adaptive optics coronagraphs, which potentially translates to a proportional savings in cost. The schematic system is shown in Figure 1. Also note that such an instrument could co-exist with a coronagraph since it uses the near on-axis light that the coronagraph discards at its central field occulter.

Advances in nulling technology enable this approach. The SIM testbed nuller, has demonstrated $99.999 \%$ stabilized nulls and $99.9999 \%$ transient nulls, and has come very close to what is needed for planet detection in space. A further 


\section{Adjustable Resolution and Diffraction \\ Control: Achromatic Nulling and}

(Shearing) Interferometer

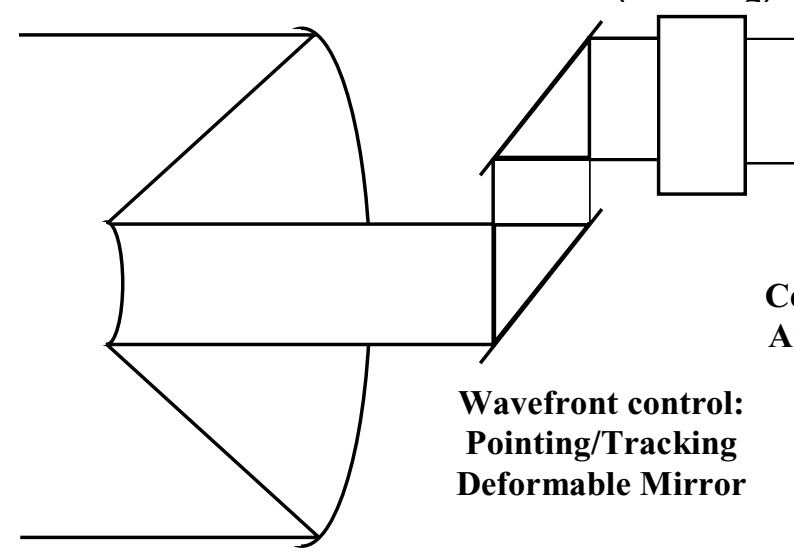

Single aperture telescope

Figure 1: General layout for planet finding using a nulling interferometer and fiber-optic array.

key element of the nulling approach is the use of single mode fiber spatial filter in conjunction with the nulling interferometer. This combination makes very deep nulling possible without the requirement to achieve and maintain $\lambda / 4000$ wavefront quality over a (large) full aperture.

\section{Science justification for future missions}

Over 100 planets around nearby stars have already been discovered by radial velocity techniques (RV). RV methods can measure the planet's orbital period, radius, eccentricity, and $M \sin (i)$. Using this concept, direct detection provides the next layer of information. Such an Optical Planet Discoverer (OPD) planet detection instrument would demonstrate the scientific feasibility of this approach using a modest sized aperture, for detection and spectroscopy of Jupiter-like planets (Mennesson et. al., 2002b). It would also demonstrate technical feasibility as well, because it would observe in the same band (optical), using the same basic instrument design, and same observing method, as the 'ultimate' visible TPF (VTPF) instrument for the detection of earth-like planets (Shao et. al., 2002). Scientific 'test targets' are already known. As we discuss later, several of the extrasolar planets already known from radial-velocity monitoring have planets should be readily detectable. Providing a low-resolution spectrometer is a highly valuable feature even if spectroscopy can be achieved only for the brightest detections thus demonstrating the first physical characterization of the atmospheres (or surfaces) of planets beyond our own solar system. Currently, an IR interferometer TPF concept would detect the 11- $\mu \mathrm{m}$ ozone feature in an exo-Earth's atmosphere. But visible spectroscopy of an exo-Earth is equally fascinating and complementary to an infrared mission. A visible nulling interferometer behind a 4-m class telescope could also search 100 's of nearby stars within $15 \mathrm{pc}$ of the Sun for Earth-like planets in the habitable zone. This is consistent with the long-term science objective to study exo-planets in both the visible and IR spectral bands.

If we view our solar system from a large distance, Earth appears only 6.6 times fainter than Jupiter. Since our detection sensitivity is limited by scattered starlight, even if a ' $47 \mathrm{UMa}$ ' planet had a density as high as Earth's (unlikely), it would still be readily detectable. Several others of the currently known extrasolar planetary systems, such as Ups And d, 16 Cyg b, and HD 160691, have orbit sizes in the peak sensitivity range for OPD. For stars closer than $10 \mathrm{pc}$, our peak sensitivity lies in the $\sim 1$-AU range. Note however, that these are all long-period planets, with $\mathrm{P}>\sim 2$ years. These estimates demonstrate that detection of Jupiters is well within the reach of OPD, and that with longer integration times, small-diameter planets should also be detectable.

\section{What can be determined from low-resolution spectroscopy}

Low-resolution $(R=\bar{\lambda} / \Delta \lambda \sim 20)$ spectroscopy of resolved planets in the spectral region 500-1000 nm enable critical additional physical and chemical characterization. Even qualitative information can be obtained from relative reflectivity spectra without requiring an absolute albedo scale. These additional properties will impose valuable constraints on models for planetary formation and evolution.

A straightforward example is differentiating between gas giant and terrestrial planets in our Solar System (Figure 2). These spectra are strikingly different: even with low SNR, and modest spectral resolution $(R=20)$ these spectra would be easily separable with OPD. It is easy to distinguish terrestrial and giant planets using $500-1000 \mathrm{~nm}$ spectra, because of the unmistakable absorption bands of methane $\left(\mathrm{CH}_{4}\right)$, the most prominent of which is at $890 \mathrm{~nm}$, evident in Jupiter and Neptune.

The absence of spectroscopic methane does not necessarily imply that the body is a terrestrial planet. Figure 2 also shows a spectrum for an extrasolar giant planet (EGP) (Sudarsky et al. 2000), representing one of the hottest models of a 
suite ranging from Jupiter-like to 'roasters'. This particular EGP model illustrates absorption features of $\mathrm{CH}_{4}$ and $\mathrm{H}_{2} \mathrm{O}$ between 890 and $1000 \mathrm{~nm}$, as well as electronic transitions of ionized Na near $700 \mathrm{~nm}$ and $\mathrm{K}$ near $800 \mathrm{~nm}$. Electronic features and the presence of $\mathrm{H}_{2} \mathrm{O}$ form a useful 'thermometer' to gauge the high-temperature end of possible EGP conditions. In other models, the presence of gaseous absorption features is partially obscured by high-level clouds which diminish the effective optical path length. These high-temperature spectra might be expected of gas giants located extremely close to their primaries.

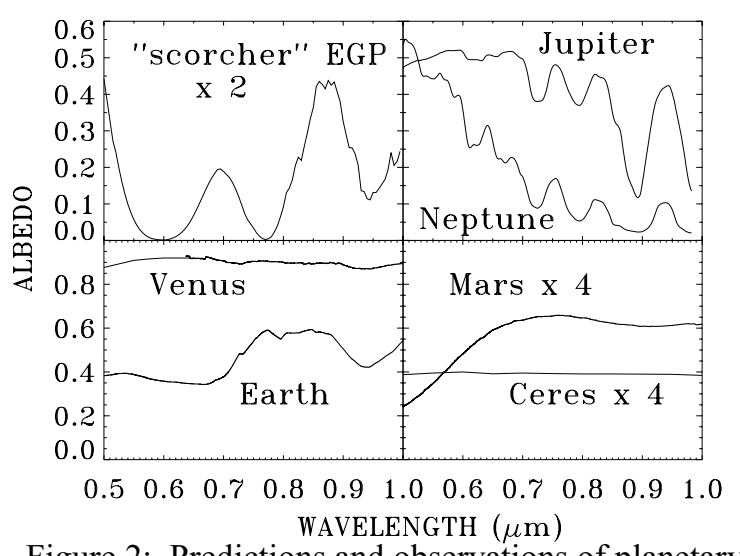

Figure 2: Predictions and observations of planetary spectra for visible light.
The spectrum of the Earth shows evidence of atmospheric scattering in the rise of the spectrum going from 0.6 to $0.5 \mu \mathrm{m}$, and also shows two of the critical signatures in NASA's search for evidence of life:

(i) water vapor absorption bands at $0.92 \mu \mathrm{m}$ (with fainter ones at 0.72 and $0.82 \mu \mathrm{m})$, and

(ii) a narrow band of $\mathrm{O}_{2}$ near $0.76 \mu \mathrm{m}$ that might be resolved above the signal level.

(iii) a spectral "vegetation edge" rising from 0.68 to $0.75 \mu \mathrm{m}$ which is due to absorption by green foliage. One could envision re-visiting a planet showing water, oxygen and hints of vegetation for extra signal, leading to confirmation of this feature.

An exciting prospect for the full scale VTPF mission, if not for OPD itself, would be to search the highest SNR detected planets for spectral variability due to rotation. If large variations in, say, cloud cover or depth exist, they should be detectable.

Planetary system inventory, demographics and planetary system dynamics

The fraction of $\mathrm{G}$ and $\mathrm{K}$ stars with Jupiters is already fairly well established at $\sim 7 \%$, for radii less than a few AU, and masses greater than $\sim 0.5$ Jupiters. But what fraction of early-type stars has 'Jupiters', relative to late-type? Prior to OPD, this may be partially answered by the French stellar photometry mission COROT (with very limited statistics) or with microlensing. COROT will be limited to short-period systems $(P<50$ days $)$, while OPD would sample planets with larger orbit radii.

OPD will allow us to explore a range of stellar types. Unlike precision RV studies, direct detection will work for any stellar type. The contrast ratio is not, to first order, a function of the stellar magnitude. By studying different stellar types, we can learn many things: How is the mass in planets related to the star's mass? Is the distribution of planetary masses and orbit radii (for the largest one or two planets in each system) a function of stellar type? How does a star's age, or evolutionary state, affect its planetary system?

Massive planets in long-period orbits are believed to stabilize the inner planets, based on our own solar system, and Nbody simulations (Levison et. al. 2001). OPD will find such systems, if they exist, and hence form target list for VTPF. These are mostly not the systems detected by current RV programs. For instance, the 51-Peg systems are not conducive to searches for Earths, because the formation and evolution of such systems appears to preclude the presence of an Earth in a circular orbit potentially stable for a billion years. Likewise, the systems with Jupiter's at larger radii show eccentric orbits, and a significant fraction are probably interacting strongly. Secular resonances are seen in HD 83443 (Wu \& Goldreich 2001); and GL 876 (Lee \& Peale 2001). OPD will provide crucial data by measuring the orbit inclinations and longitudes of ascending nodes. Even without accurate masses, these data would allow very detailed simulations of longterm system stability, which are important for the longevity of Earths. OPD will push to lower mass planets; as the role of Neptune-mass planets in stabilizing an inner-planet zone is currently unknown.

For planets detected in short-period orbits $(<1-2$ years) we can study dynamics, by tracing enough of the orbits to solve for Keplerian parameters. Orbit fits must include illumination phase, hence allowing an estimate of the $100 \%$ illumination brightness. In particular, since we are constrained to orbit radii less than a few AU, we will be in the sensitivity range of the RV studies. OPD complements the ground-based radial velocity work, by detecting planets at similar orbital radii. Combining RV data with detection by OPD provides a complete picture of the planetary system: all 7 orbital elements; planetary mass, and (for an assumed albedo) radius and density also.

\section{Nulling System Design}

The most challenging technological problems are those concerning suppression of starlight in the resulting image. Deep stellar nulling requires a broadband, stable subtraction scheme, so the residual limitations inherent in earlier designs need to be overcome. These limitations have been identified, and can be largely obviated simply using a reversed pair of beam splitters. Additional suppression is gained by filtering off-axis, high spatial frequency star light. This has been 
successfully used in the past in interferometry (Serabyn et.al., 1999), and has recently been suggested in the literature for planet detection (Mennesson, Ollivier \& Ruilier 2002a).

\section{Nulling Description and Interferometer Layout}

As part of the earlier SIM technology development effort, an optical nulling testbed was developed at JPL. The approach used was that of an achromatic field flip introduced between the two incident beams by means of a rotational shearing interferometer (Shao and Colavita 1992). Although this nuller met and even exceeded the nulling performance requirements set for the SIM mission, its requirement was eliminated from the mission. With laser sources, transient nulls exceeding 1,000,000:1 and stabilized nulls of 100,000:1 were obtained, while with a broadband thermal source, broadband radiation of $18 \%$ bandwidth was stably nulled to the 10,000:1 level, with transient nulls of up to 70,000:1 seen (Wallace et. al., 2000). Several limitations have been further identified in the approach. Residual asymmetries in the interferometer layout limited the ultimate null depths attainable: unequal numbers of anti-reflection-layer traversals, unequal reflections from opposite sides of the beamsplitter coating, and polarization asymmetries in the beamsplitter. All have been eliminated in the current conceptual design. A further important simplification arises if one considers a separation of the field-flip and beam-combination stages. These considerations led to the emergence of a fully symmetric nuller design (Serabyn and Colavita 2001) using a simple constructive Mach-Zehnder-like beam combiner. Because of the complete symmetry of this design, substantially improved performance is expected, allowing even deeper and more broadband nulls, as well as dual-polarization nulls. A related modified Mach-Zehnder approach is being developed in the thermal infrared for use in the Keck Interferometer nuller. For this mission, we have chosen the single input versions as differentiated from designs used to combine light from two physically separate arms of an interferometer (Figure 3). Our principal concept is to synthesize a 4 element interferometer from the telescope pupil arranged in an 'Angel cross'(Angel, 1990). This is accomplished with two nulling interferometers (Shao, 1990, Serabyn and Colavita, 2001) that also shear the pupil. With coupling optics between the two nullers that rotates the pupil by $90^{\circ}$, the final output pupil looks like Figure 4. The resulting nulled output is then spatially filtered by an array of optical fibers. The shear distance provides the interferometer baseline, the nulling cancels the starlight by destructive interference, and the fiber array spatially filters the scatter yet preserves the pupil geometry. An imaging system transforms the resultant nulled pupil into either an image or a spectrum.

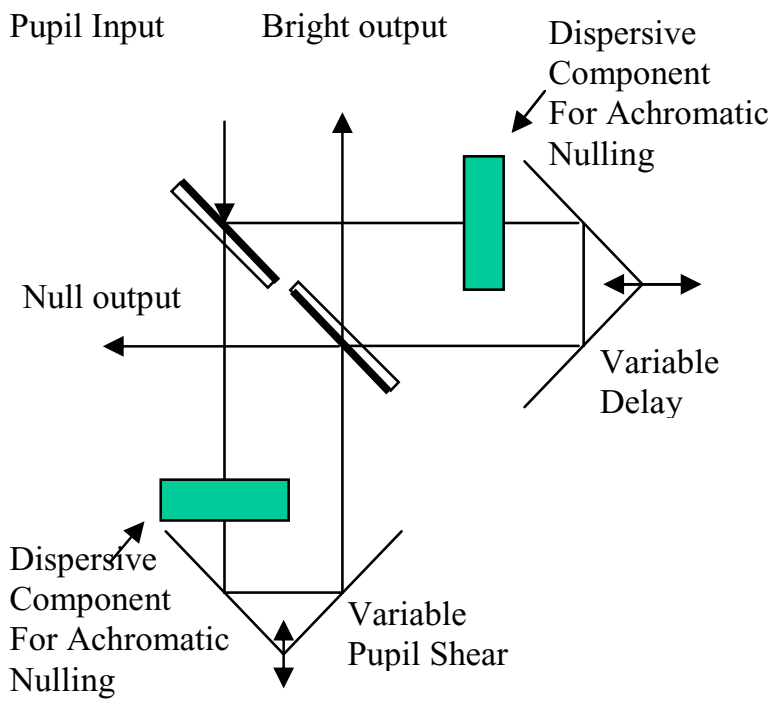

Figure 3: Nulling interferometer layout.

\section{Performance and sensitivity considerations Required null depth $\mathbf{N}$.}

Whereas a conventional 2 beam interferometer with a projected baseline, $b=b_{x}=b_{y}$, and with wave number, $k=2 \pi / \lambda$ produces a nulled fringe intensity proportional to $\theta^{2}$, where $\theta$ is the angle from the optical axis,

$I \propto(k b)^{2} \theta^{2}$ 
the 4 subapertures produce an intensity proportional to $\theta^{4}$ thus deepening the null from the star and enhancing the contrast between the nulled star and the reflected planetary light (Angel and Woolf, 1997, Mennesson and Mariotti, 1997):

$I \propto(k b)^{4} \theta^{4}$.

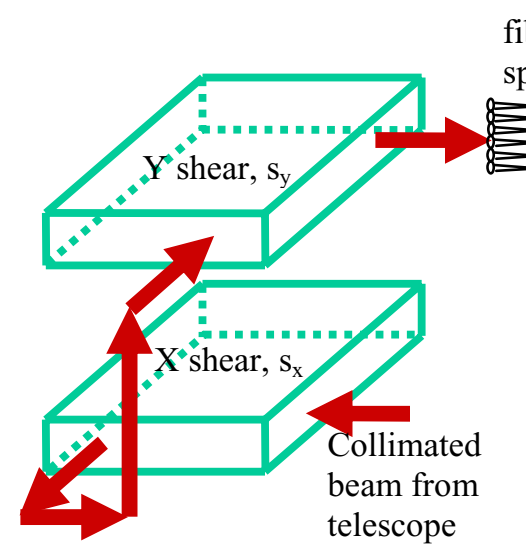

Turning/Rotation Mirrors fiber-optic array spatial filter

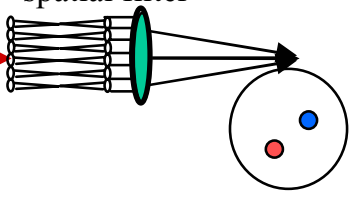

Image plane (real image)

Figure 4: Pupil shear pattern with synthesized baselines $b_{x}$ and $b_{y}$, and image formation using two nulling interferometers.

The aditional angular suppression is needed to provide a sufficiently deep null over the spatial extent of a star similar to the sun (1mas). This pattern is shown in Figure 5 below for both a narrow and extended fields of view.
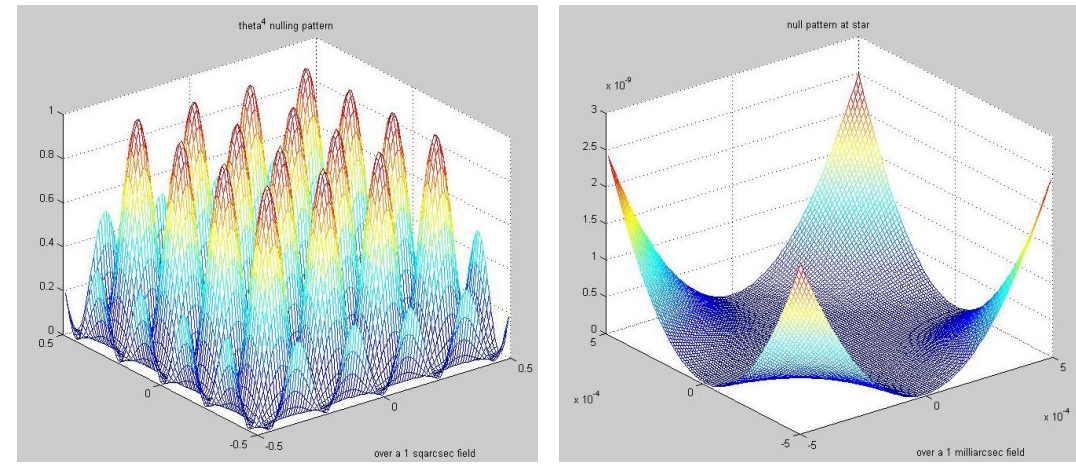

Figure 5: Intensity response of a four element nulling inteterferometer. Left, fringe pattern over a 1 as field of view. Right, nulling depth over 1 mas, the angular extent of a sun at $10 \mathrm{pc}$.

Further starlight suppression is accomplished by sampling the nullled subaperture by an array of microlenses and filtering the light via single mode optical fibers (Shao, 1990). Recollimation by a similar lens array provides a corrected exit pupil, and imaging by a simple achromatic lens produces the final nulled image of the planetary system. The basic idea and major advantage to this design is that no active wavefront correction is required for spatial scales smaller than the size of the lenslet. This is due to using single mode optical fiber. On the otherhand, using an array of fibers at the $5 \mathrm{~cm}$ scale at a pupil guarantees a 2 " field of view for nulling imaging. The main figure of merit for a nulling interferometer is its rejection rate. It is defined as the ratio of constructive to destructive intensity signals as obtained on a source of given characteristics (spatial and spectral extent for instance). Even for perfect pointing and perfect wavefront quality, the interferometric transmission is only zero on the line of sight, so that a star of finite angular size will always produce leaks limiting the rejection rate. This is referred as residual "stellar leaks" or limited "null depth" $\mathrm{N}$. In practice, null depths as low as $10^{-7}$ are necessary to compensate for the large contrast ratio between stellar and planetary visible fluxes.

\section{Entrance pupil shear}

In order to efficiently reject the stellar light, we propose subdividing the entrance pupil into 4 sub-apertures to be nulled out according to an Angel cross (Angel 1990) recombination scheme. Defining b as the baseline between sub-apertures centers in a pair, assuming perfect pointing, and integrating the leaks over a uniform stellar disk of full angular extent

$\theta$, the theoretical null depth $\mathrm{N}$ is given by (Mennesson et. al., 2002b):

$$
N=\underline{1536}(\pi \cdot b \cdot \Delta \theta / \lambda)^{4}
$$


Normalizing to the case of a 1 mas diameter star (a Sun at 10pc has an apparent diameter of 0.9 mas), seen at $500 \mathrm{~nm}$, with sub-apertures separated by $1 \mathrm{~m}$, we get:

$$
N=3.25 \times 10^{-11} \cdot\left(\Delta \theta_{\text {mas }}\right)^{4} \cdot\left(\frac{0.5}{\lambda_{\mu m}}\right)^{4} \cdot\left(\frac{b_{m}}{0.7}\right)^{4}
$$

Ensuring stellar leaks smaller than $5 \times 10^{-8}$ at $500 \mathrm{~nm}$ on Procyon (closest $\mathrm{F}$ star) which is about 3 mas in angular diameter requires a baseline, $\mathrm{b}$, smaller than $.7 \mathrm{~m}$. This is consistent with forming 4 sub-apertures in the 60 to $80 \mathrm{~cm}$ diameter class out of a $1.5 \mathrm{~m}$ class telescope as suggested for OPD. A full VTPF mission based on a visible nuller concept uses a larger primary mirror, typically $4 \mathrm{~m}$ in diameter would need only a relatively small amount of shear so that the closest stars are not too resolved (Shao et. al., 2002).

\section{Achromatic Nulling Beam Combiner}

The symmetric nuller as currently used does have one limitation for our application; that being that a field flip is accompanied by an aperture inversion. For the sub-aperture sampling described here, this results in undesirable shifts in location of the combining subapertures. To avoid this, it is necessary to introduce the necessary achromatic $\pi$-radian phase shift in a different fashion, such as pairs of dielectric plates of differing thicknesses. Solutions for achromatic $\pi$ radian phase shifts (to the needed accuracy) exist with two glasses. Thus for this experiment, the final layout for the beam combiner consists of identical two-glass pairs of (rotatable) dielectric phase retarders (Morgan et. al. 2000, Morgan 2002), in each leg of the interferometer. Note that the combination of common BK7 and Fused Silica optical glasses the theoretical minimum of $10^{-7}$ can be realized (Figure 6). This design will be verified in laboratory experiments.

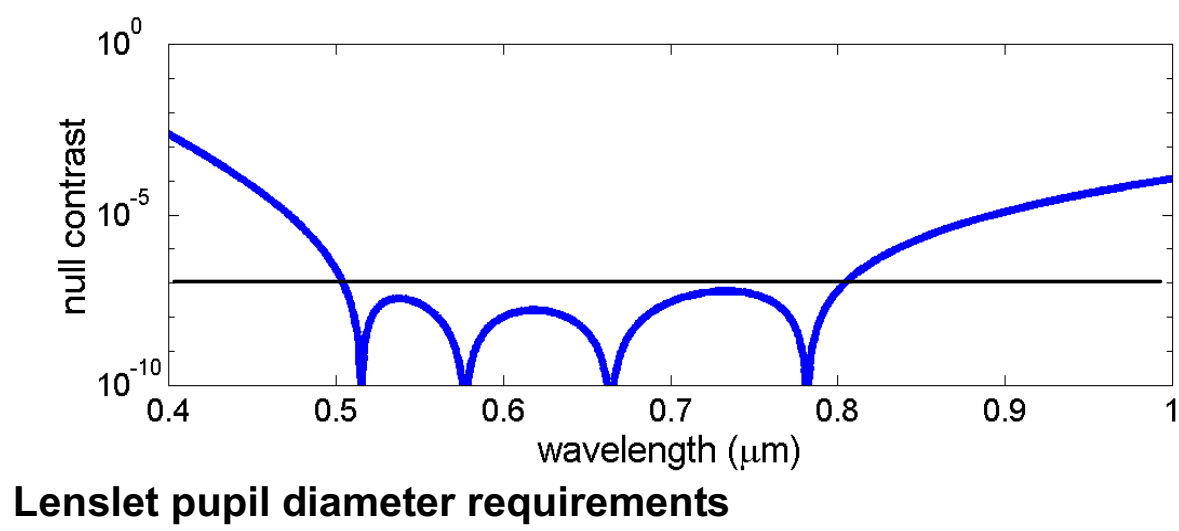

Figure 6: Calculation for nulling depth for common optical glasses, BK7 and Fused Silica.

The injection of a stellar wavefront into a single-mode fiber is optimized for an f-ratio that matches the Airy spot and fiber core sizes. If only one common fiber were used to clean the wavefront, the field of view would then be limited to a sub-aperture Airy pattern. In order to keep some reasonable field of view together with the advantages of spatial filtering, the nuller output pupil is subdivided in sub-pupils of diameter $d$, which are imaged onto an array of single-mode fibers via a lenslet array. The accessible field of view is now $\lambda / \mathrm{d}$, so that lenslet pupils smaller than $5 \mathrm{~cm}$ guarantee a $2 \operatorname{arcsec}$ diameter field of view at any $\lambda>500 \mathrm{~nm}$. This is enough to detect Jupiter analogs around the closest main sequence stars. Using sub-apertures diameters ranging from $60 \mathrm{~cm}$ to $80 \mathrm{~cm}$, an array of 100 to 200 fibers is needed. Imaging of Earth-like planets will require the order of 1000 subapertures.

\section{Single-mode fibers}

The single-mode fiber filtering effect allows us to get rid of all wavefront distortions at the $5 \mathrm{~cm}$ lenslet pupil size level, and smaller. Single-mode fibers basically trade all local amplitude and phase mismatches (besides piston) between the recombined wavefronts against global intensity mismatches. Intensity mismatches are of secondary effect on the null depth. Thanks to this property, the instrumental null depth no longer varies as $\sigma_{\varphi}^{2}$ the wavefronts initial phase variance, but as $\sigma_{\varphi}^{4}$ (Mennesson et al. 2002a), or even better if active intensity correction is implemented. For a given wavefront amplitude and phase profile, much deeper stellar light cancellation is then achievable when single-mode waveguides are used (Shao 1991). Single-mode fibers also have the advantages of broad-band utilization; the injection efficiency is roughly achromatic over an octave, and perfect amplitude profile matching to the wavefronts to be nulled out. Polarization maintaining silicon based single-mode fibers are well developed in the visible to near infrared wavelengths and widely used in observational astronomy.

\section{Nulling requirements}

The fraction of stellar photons leaking through the final focal plane at the planet's image location, needs to be kept below $10^{-8}$ for sensitivity reasons. Residual stellar light is expected to be inccoherent with random phase fluctuations about zero after its path through the array of single-mode fibers so that its energy distribution is uniformly distributed on the focal 
plane. Owing to this spatial dilution effect, rejection ratios around $10^{7}$ (null depths of $10^{-7}$ ) will be enough to reach the above $10^{-8}$ level for any planet located further than 150mas away from the star.(Mennesson et. al., 2002b)

\section{Imaging optics}

Once the stellar light has been cancelled at the $10^{-7}$ level in the array of fibers, requirements for downstream imaging optics are considerably relaxed. Fiber lengths and differential pistons between lenslet pupils need to be matched within $\lambda / 20$ (but with higher stability) to ensure sufficient image quality and stability in the final focal plane. In extreme cases it may be necessary to include an imaging deformable mirror to correct large piston errors between sub-pupils. Observations with the visible nuller are made by either a CCD camera or a spectrometer. A movable mirror could allow us to choose between the two instruments.

\section{Low resolution spectrometer}

Once planets have been detected and located with maximum sensitivity, a movable mirror sends the scientific beam towards a spectrometer. Two types of spectrometers are being considered, one is a "long" slit spectrometer, where the slit direction is aligned in the direction of maximum planet throughput. The second is an integral field spectrometer that would measure the spectra of every object in the field.

In the latter case, an image is formed on a 2-dimensional bundle array of 1500 multi-mode fibers. These are rearranged along one direction to allow spectral dispersion in the perpendicular one. A final CCD matrix using 1500 pixels in the spatial dimension and 15 in the spectral one is enough to carry-out integral field low resolution (20) spectroscopy in the $500-1000 \mathrm{~nm}$ range.

\section{Performance Studies}

\section{Integration times for broad-band planetary detection and spectroscopy}

Broadband planetary detection would be carried out over the whole $500-1000 \mathrm{~nm}$ spectral range. We derive in the following the integration times necessary to detect Jupiter and Earth analogs around nearby stars of various types, using respectively a $1.5 \mathrm{~m}$ telescope (OPD) and a $4 \mathrm{~m}$ extended capability (VTPF). The terms in the integration time formula are as follows: Telescope diameter, $D$, is $1.5 \mathrm{~m}$ for OPD and $4 \mathrm{~m}$ for VTPF, eff, the overall optical efficiency (including primary area filling factor, optics transmission and detector quantum efficiency) is $15 \%$, interferometric off-axis point-source transmission is $25 \%$. The fraction of stellar leakage, sl, at planetary location is $10^{-8}$. Dist is the stellar distance in pc, Reso $=\lambda / \Delta \lambda=700 / 500=1.4$, the spectral resolution. Sep, is the star-planet separation in au, and $F$ denotes the appropriate body flux. The integration times for planet detection are:

$$
T_{\text {int }}=\left\{\begin{array}{l}
7 \min \left(\frac{S N R}{5}\right)^{2}\left(\frac{1.5}{D(m)}\right)^{2}\left(\frac{0.15}{e f f}\right)\left(\frac{s l}{10^{-9}}\right)\left(\frac{d i s t}{10(p c)}\right)\left(\frac{\operatorname{Re} s o}{1.4}\right)\left(\frac{S e p}{5.4}\right)^{4}\left(\frac{F_{\text {sun }}}{F^{*}}\right) \text { Jupiters } \\
7.7 h r\left(\frac{S N R}{5}\right)^{2}\left(\frac{4.0}{D(m)}\right)^{2}\left(\frac{0.15}{\text { eff }}\right)\left(\frac{s l}{10^{-9}}\right)\left(\frac{d i s t}{10(p c)}\right)\left(\frac{\operatorname{Re} s o}{1.4}\right)\left(\frac{S e p}{5.4}\right)^{4}\left(\frac{F_{\text {sun }}}{F^{*}}\right) \text { Earths }
\end{array}\right.
$$

Figure 7 summarizes the required integration times for detection of Jupiter and Earth analogs around stars of variable spectral types located $10 \mathrm{pc}$ away, with nominal values for the observing parameters as noted. The integration times were all computed as a function of solar flux and reflected flux, planet albedo, and stellar leakage. Once a planet has been detected, telescope rotation angle may be chosen to place it on a constructive fringe and carry out spectroscopic analysis with optimized sensitivity around a given wavelength (4x increase in signal, Shao et. al., 2002 and Menneson et. al, 2002b). For a spectrograph with $\mathrm{R} \sim 20$, the increase in exposure time is only 5 .
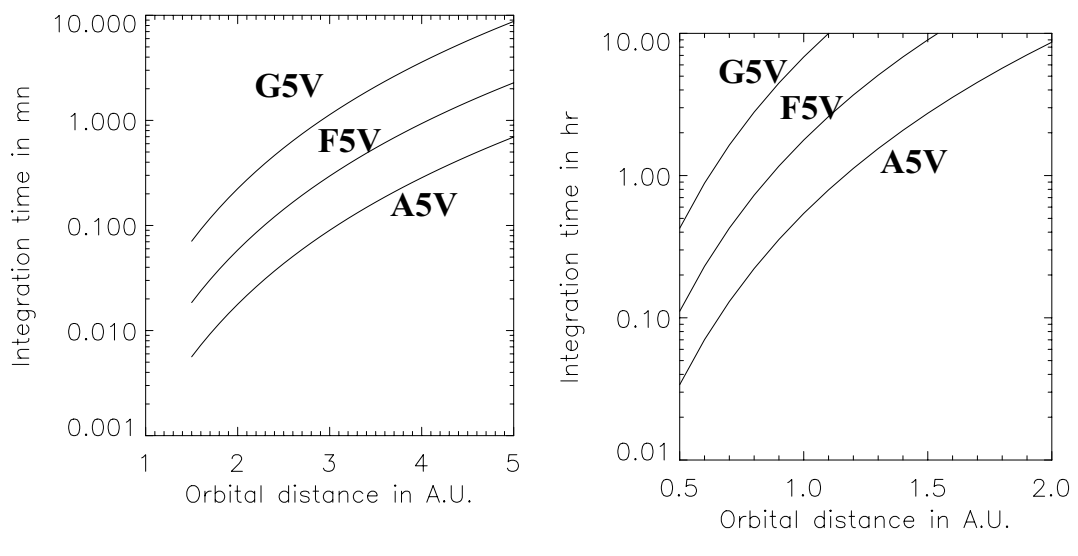

Figure 7: Integration times for $5 \sigma$ detection of planet analogs at $10 \mathrm{pc}$ around main sequence stars of various spectral types. Left: Jupiter-like planets using a $1.5 \mathrm{~m}$ telescope, Right: Earthlike planets using a $4 \mathrm{~m}$ telescope. 
The response pattern of the nuller-fiber array combination is shown in Figure 8. The left hand figure is the same pattern shown in Figure 5, but the right hand figure is the response integrated over all wavelengths and angular and orientations. It shows the maximum sensitivity at $\lambda / 2 b$ radius while suppressing sensitivity at the longer radial distances. Future data processing that is not dependent on such a wide spectral bandpass may enable advanced detection at extended radial distances.

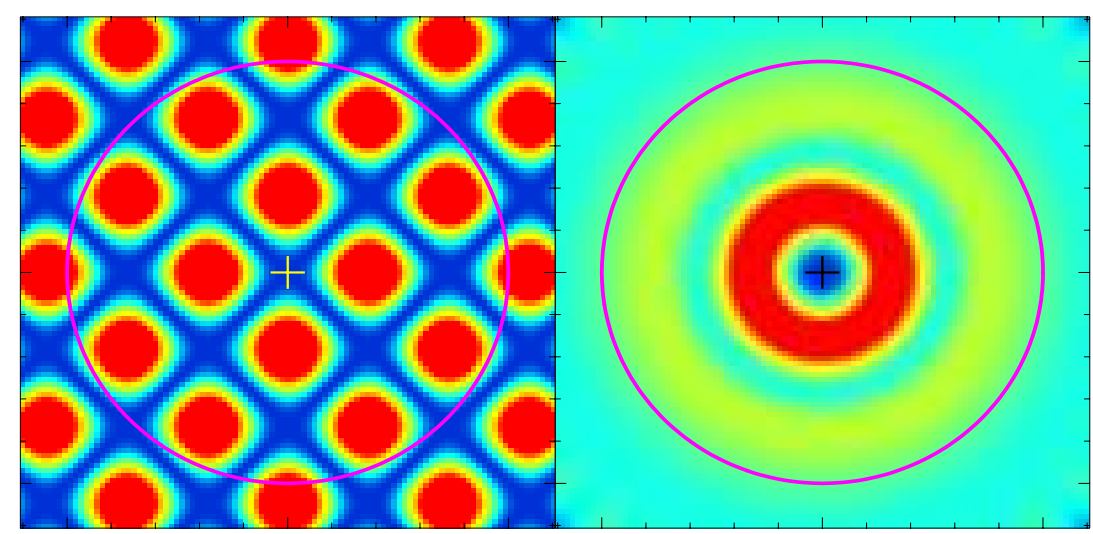

Figure 8: The null response pattern of a $1.5 \mathrm{~m}$ precursor. (left) at wavelength $650 \mathrm{~nm}$ and at one orientation. (right) Using wavelengths $500-1000 \mathrm{~nm}$ and combining all orientations $0-360 \mathrm{deg}$. The circle represents $5 \mathrm{AU}$ radius at $10 \mathrm{pc}$ distance. Response maxima are shown in red .

Figure 9 shows the brightness of the planets and the effect of the stellar leakage in the image for a star at 10pc and for a nearby star e-Eri (3.3pc). Null depth of $10^{-7}$ was assumed with 100 fibers for OPD and 1000 fibers for the VTPF configuration. Note that the planet fluxes are averaged over all orientations of the null pattern. Spectral studies will be made at a single orientation at which the observed flux can be much higher. The effects of the stellar leakage, therefore, are not a serious problem.

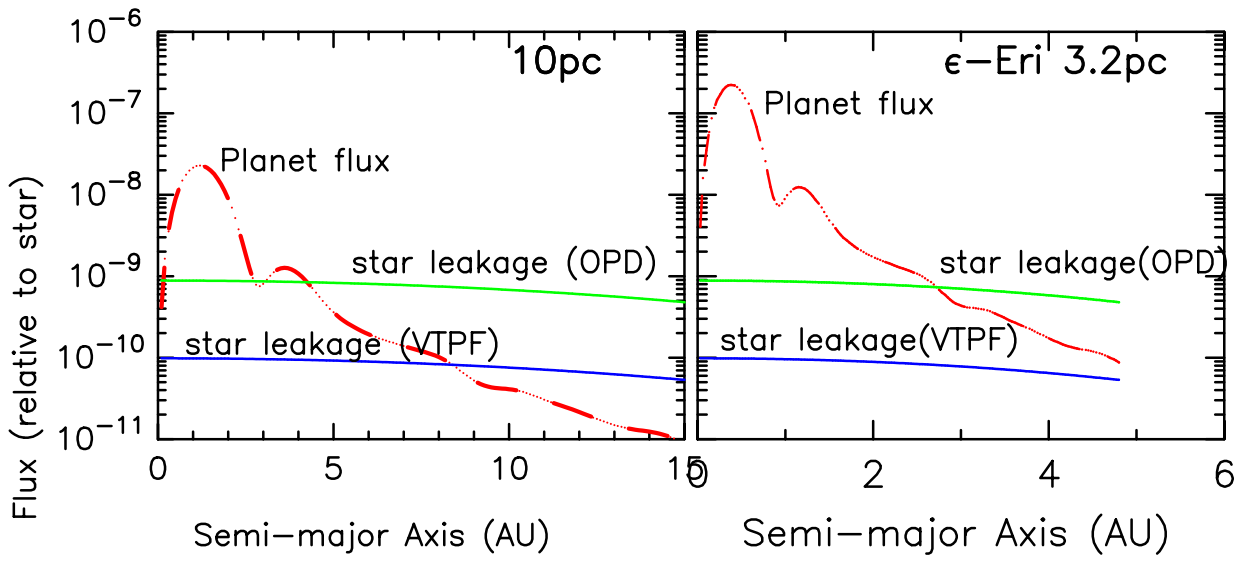

Figure 9: Observed planet fluxes compared to the PSF of the stellar leak as a function of the planet distance. The planet flux densities are assumed to be that for Jupiter and given in units of stellar flux.

Finally in Figure 10 we show simulated image of a solar system analog at a distance of $10 \mathrm{pc}$ as observed by OPD and by VTPF. OPD is capable of resolving Jupiter. For VTPF, even using a conservative $10^{-7}$ null, the high spatial resolution $(\sim$ $20 \mathrm{mas})$ and dynamic range $(>1000)$ in the image allow us to detect and study Earth-size planets in the habitable zones around stars at 10pc. In the simulated image Venus, Earth, Jupiter and Saturn are clearly detected.

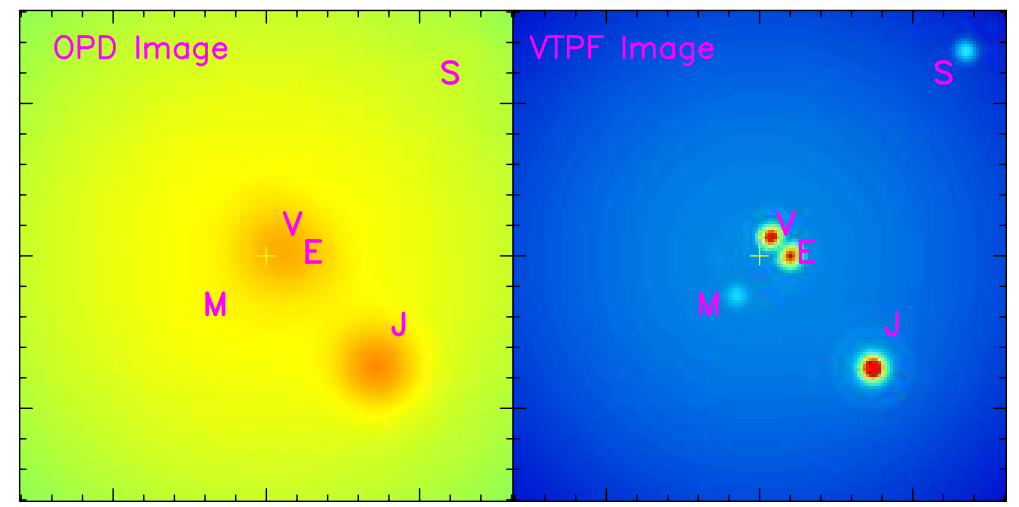

Figure 10: Simulation of a solar system analog at 10pc demonstrating OPD and VTPF imaging capability. (left) OPD image (right) VTPF image The brightest emissions are colored red. 


\section{Summary}

The detection of planets outside of our solar system with a space telescope can be accomplished with modestly sized telescope by using a nulling interferometer in the pupil plane of the telescope followed by a coherent array of fiber optic spatial filters. Simulation experiments show that a Jupiter like planet can be detected with a $1.5 \mathrm{~m}$ diameter telescope, and an Earth like planet can be detected with a $4 \mathrm{~m}$ telescope. The difficulty in full aperture wavefront control has been transferred to the nulling interferometer whose capabilities has been already established in previous technology demonstrations, and also eliminates extreme optical surface requirements on the primary mirror at the mid spatial frequencies. A symmetric nulling interferometer adjustable in spatial shear has been designed and is being fabricated. Laboratory experiments are underway to verify this design and to verify its performance as well as that for a coherent optical fiber array.

\section{Acknowledgement}

This work was preformed at the Jet Propulsion Laboratory, California Institute of Technology, under contract to the National Aeronautics and Space Administration.

\section{References and Bibliography}

Angel, R., (1990), "Use of a 16-m Telescope to Detect Earthlike Planets," Proceedings of the Workshop on The Next Generation Space Telescope, P. Bely and C. Burrows, eds., Space Telescope Science Institute, pp. 81-94.

Angel, J.R.P, and Woolf, N.J., (1997),"An Imaging Nulling Interferometer To Study Extrasolar Planets,"Astrophysical Journal, v475, pp. 373-379.

Gaffey, M. J., J. F. Bell, and D. P. Cruikshank, (1989), Reflectance spectroscopy and asteroid surface mineralogy. In Asteroids II, Binzel et al., eds., U. Arizona Press, Tucson, pp. 128-147.

Karkoschka, E., (1994), Spectrophotometry of the Jovian planets and Titan at 300-nm to 1000-nm wavelength: The methane spectrum. Icarus, v111, 174-192.

Lee, M. \& Peale S., (2001), Evolution of the GJ876 Planets into the 2:1 Orbital Resonance. BAAS, 34, 3.03

Levison, H. F., Dones, L., Canup, R., Agnor, C., and Duncan, M. J., (2001), The Role of Giant Planets in Terrestrial Planet Formation. In 32nd Annual Lunar and Planetary Science Conference, March 12-16, 2001, Houston, Texas, abstract no. 1232.

Malbet, F.,Yu, J., Shao, (1995), M., Pub Astron Soc Pacific, V107, pp. 386-398, April 1995.

Marley, and C. M. Sharp, (2000), The near-infrared and optical spectra of methane dwarfs and brown dwarfs. Astrophys. J. v531, pp. 438-446.

Meadows, V. et al., (2000), Astronomical detection of biosignatures from extrasolar planets, JPL/Caltech proposal to the NASA Astrobiology Institute CAN-00-OSS-01.

Menneson, B., and Mariotti, J.M., (1997), "Array Configurations for a Space Infrared Nulling Interferometer Dedicated to the Search for Earthlike Extrasolar Planets", Icarus, v128, pp. 202-212.

Mennesson B.,Ollivier M., and Ruilier C., (2002a), "On the use of single-mode waveguides to correct the optical defects of a nulling interferometer", J. Opt. Soc. Am. A, Feb 2002.

Mennesson, B.P., Shao, M., Levine, B.M., Wallace, J.K., Liu, D.T., Serabyn, E., Unwin, S.C., Beichman, C.A., (2002b), 'Optical Planet Discoverer: how to turn a $1.5 \mathrm{~m}$ class space telescope into a powerful exo-planetary systems images', SPIE, v4860.

Morgan, R., Burge, J., and Woolf, N., (2000), "Nulling Interferometric Beam Combiner Utilizing Dielectric Plates" Experimental Results in the Visible Broadband SPIE v4006.

Saumon D., Hubbard W.B., Burrows A. et al., (1996), ApJ 460, 993.

Serabyn, E., Wallace, J.K., Hardy, G.J., Schwindthin, E.G.H., and Nguyen, (1999), “Deep Nulling of Visible LASER Light”, Appl. Opt., v38, p7128.

Serabyn, E. and Colavita, M.M., (2001),'Fully Symmetric Nulling Beam Combiners', Applied Optics, v40, pp. 16681671.

Shao, M.,(1991), "Hubble Extra Solar Planet Interferometer", SPIE v1494.

Shao, M., Serabyn, E., Levine, B.M., Mennesson, B.P., and Velusamy, T, (2002), 'Visible nulling coronagraph for detedting planets around nearby stars', SPIE v4860.

Sudarsky, D., A. Burrows, and P. Pinto. (2000), Albedo and reflection spectra of extrasolar giant planets. Astrophys. J. v538, 885--903.

Wallace, K., Hardy, G, and Serabyn, E., (2000), "Deep and stable interferometric nulling of broadband light with implications for observing planets around nearby stars", Nature, v406.

Wu, Y., and Goldreich, P., (2001), Tidal evolution of the planetary system around HD 83443. Astrophys. J. submitted 